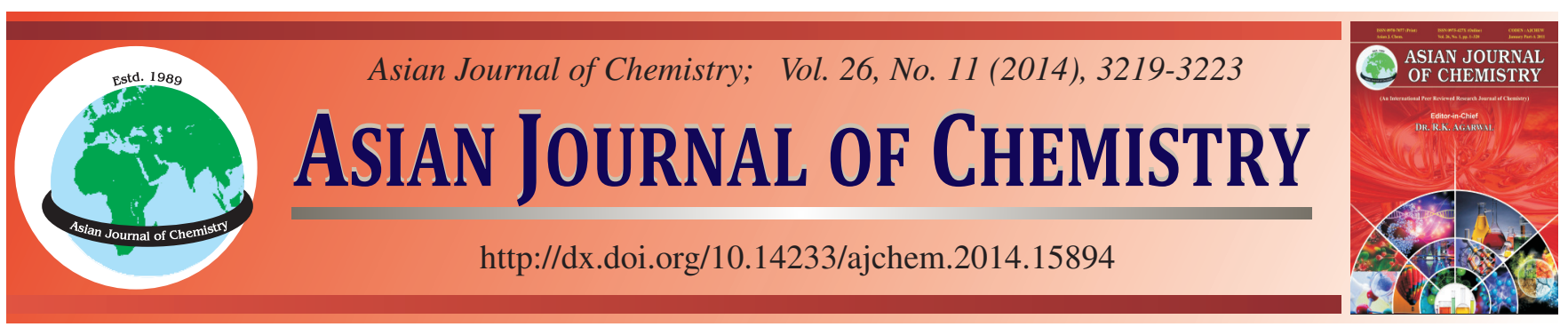

\title{
Concise Syntheses and Antitumor Activities of $\alpha$-Hydroxy(mercapto)amide Derivatives
}

Y.X. LI* and Z. QIU

Chemistry and Chemical Engineering Department, Huaihua University, 612 Yingfeng Road, Huaihua 418008, P.R. China

*Corresponding author: E-mail: hhxyyxli@163.com

Received: 11 June 2013;

Accepted: 17 October 2013;

Published online: 25 May 2014;

AJC-15217

A novel process for preparing $\alpha$-hydroxy(mercapto)- $N$-[6-(3-phenylureido)hexyl]amide derivatives was described and three new compounds 8-10 were synthesized. The antitumor activities on Hut102, MCF7 and HepG2 of the compounds 7-10 were assayed. The results showed that the target compounds 7-10 exhibited some antitumor activities against tumor cell lines.

Keywords: Histone deacetylase inhibitors, $\alpha$-Hydroxy(mercapto)amide, Drug design, Antitumor activity.

\section{INTRODUCTION}

Histone acetylation and histone deacetylation are essential for chromatin remodeling and regulation of gene transcription in eukaryotic cells. Histone deacetylases and histone acetyltransferases are two classes of enzymes that catalyze the deacetylation and acetylation of lysine residues located in the $\mathrm{NH}_{2}$ terminal tails of core histones ${ }^{1,2}$. Perturbations of this balance in the form of histone deacetylation are often observed in human tumors ${ }^{3}$. Aberrant activation of histone deacetylases results in the transcriptional repression of oncoprotein and is linked to the malignant phenotypes of tumors ${ }^{4}$. Thus, Histone deacetylase inhibitors have become promising anticancer agents in recent years. They have shown ability to block angiogenesis and cell cycling, as well as initiate differentiation and apoptosis ${ }^{5-6}$. At present, many of the histone deacetylases inhibitors such as suberoylanilide hydroxamic acid (SAHA), trichostatin A (TSA), Panobinostat (LBH 589) and MC 1568 have been reported (Fig. 1a) and the histone deacetylases inhibitors have potent anticancer effects in vitro and in vivo ${ }^{7-8}$. Among them, in 2006, Zolinza (SAHA, vorinostat) became the first histone deacetylases inhibitor to acquire FDA approval and is used for the management of the cutaneous manifestations of T-cell lymphoma ${ }^{9}$. On the whole, the classic pharmacophore<smiles>CC(/C=C/C(=O)NO)=C\C(C)C(=O)c1ccc(N(C)C)cc1</smiles><smiles>CC(C)C(=O)SCCCCCCC(=O)Nc1nc(-c2ccccc2)cs1</smiles>

Fig. 1. Small molecule histone deacetylases inhibitors; (a) Selected examples of histone deacetylases inhibitors; (b) Pharmacophoric model of histone deacetylases inhibitors; (c) Structures of NCH-31 and NCH-51 
for histone deacetylases inhibitors consists of three distinct structural motifs: the zinc-binding group (enzyme binding), a hydrophobic linker and a surface recognition group ${ }^{10,11}$ (Fig. 1b). Typically, the common zinc-binding group of histone deacetylases inhibitors is the hydroxamate moiety. Study on the structural modifications of the hydro-xamate zinc-binding group of histone deacetylases inhibitors has recently attracted extensive attention. Kozikowski and coworkers reveals that mercaptoacetamide-based histone deacetylases inhibitors, in which the hydroxamate moieties of SAHA enzyme binding domain were replaced by mercaptomethyl amide, would probably avoid the dose-dependent toxicity associated with the hydroxamate-based inhibitors ${ }^{12}$. Compounds containing a sulfydryl or isobutyryl sulfydryl in enzyme binding domain were both potent histone deacetylases inhibitors ${ }^{13}$ (Fig. 1c). To search for novel antitumor hits, our research was initially based on the structure of SAHA. According to the strategy of bioisosteric replacement, the surface recongnition and enzyme binding domain were modified with phenylureido and $\alpha$-hydroxy(mercapto), respectively. Herein we wish to disclose our preliminary efforts on this subject.

\section{EXPERIMENTAL}

Unless otherwise noted, reagents were purchased from commercial suppliers and used without further purification, as all solvents were redistilled before use. NMR spectra were recorded on a Mercury-Plus 400 spectrometer in $\mathrm{CDCl}_{3}$ or DMSO- $d_{6}$ with TMS as the internal reference, the chemical shifts were reported in $\delta \mathrm{ppm}$. MS spectra were determined using a TraceMS 2000 or Waters 2Q4000 LC/MS organic mass spectrometer. Elemental analyses were performed on a Vario EL III elemental analysis instrument. Melting points were measured on a Buchi B-545 melting point apparatus and are uncorrected. Purity of all compounds was checked by TLC on precoated silica gel G plates (Kiesel gel $0.25 \mathrm{~mm}, 60 \mathrm{G} \mathrm{F}_{254}$, Qingdao, China).

Synthesis of $\boldsymbol{\alpha}$-hydroxy(mercapto)amides: The title compounds were synthesized by a convenient five-steps procedure as outlined in Scheme-I.

Preparation of tert-butyl 6-aminohexylcarbamate (2) ${ }^{14,15}$ : To a solution of hexane-1,6-diamine (60 g, $\left.0.516 \mathrm{~mol}\right)$ 1 in $\mathrm{CH}_{2} \mathrm{Cl}_{2}(200 \mathrm{~mL})$ was added (Boc) $)_{2} \mathrm{O}(22.5 \mathrm{~g}, 0.103 \mathrm{~mol})$ during $0.5 \mathrm{~h}$ at $0{ }^{\circ} \mathrm{C}$, then the mixture was stirred at room temperature for $5 \mathrm{~h}$, extracted with $\mathrm{CH}_{2} \mathrm{Cl}_{2}(150 \mathrm{~mL} \times 3)$, dried with anhydrous magnesium sulfate, filtered off with suction. The combined organic layer was concentrated on a rotary evaporator to give colourless oil 2 in yield $96 \%$ (21.5 g). The product was used directly for next reaction without purification.

Preparation of tert-butyl 6-(3-phenylureido)hexylcarbamate (3) ${ }^{\mathbf{1 6 , 1 7}}$ : To a solution of intermediate $2(0.5 \mathrm{~g}, 2.31$ $\mathrm{mmol})$ in $\mathrm{CH}_{2} \mathrm{Cl}_{2}(6 \mathrm{~mL})$ was added isocyanatobenzene $(0.33$ $\mathrm{g}, 2.77 \mathrm{mmol})$ at $0{ }^{\circ} \mathrm{C}$, then the mixture was stirred at room temperature for $4 \mathrm{~h}$, extracted with $\mathrm{CH}_{2} \mathrm{Cl}_{2}(50 \mathrm{~mL} \times 3)$, dried with anhydrous magnesium sulfate, filtered off with suction. The combined organic layer was concentrated on a rotary evaporator, purified by chromatography on silica using petroleum ether/ethyl acetate $(15: 1, \mathrm{v} / \mathrm{v})$ as eluent to give white solid 3 in yield $95 \%$ (0.73 g). m.p. 97-99 ${ }^{\circ} \mathrm{C} .{ }^{1} \mathrm{H}$ NMR (400 $\mathrm{MHz}, \mathrm{CDCl}_{3}$ ) $\delta$ (ppm): 1.26 (s, $4 \mathrm{H}, \mathrm{CCCH}_{2} \mathrm{CH}_{2} \mathrm{CC}$ ), 1.36 (s, $\left.9 \mathrm{H}, 3 \times \mathrm{CH}_{3}\right), 1.41\left(\mathrm{~s}, 4 \mathrm{H}, \mathrm{CCH}_{2} \mathrm{CCCH}_{2} \mathrm{C}\right), 2.88\left(\mathrm{dd}, J_{1}=6.4\right.$<smiles>CC(C)(C)OC(=O)NCCCCCCN</smiles><smiles>CCOC(=O)C(C)(C)C(C)(C)OC(=O)NCCCCCCNC(=O)Nc1ccccc1</smiles><smiles>CC(S[13OH])C(=O)NCCCCCCNC(=O)Nc1ccccc1</smiles>

7: $\mathrm{R}=\mathrm{H} ; 8: \mathrm{R}=\mathrm{CH}_{3}$<smiles>[R]C(Cl)C(=O)NCCCCCCNC(=O)Nc1ccccc1</smiles>

5: $\mathrm{R}=\mathrm{H} ; 6: \mathrm{R}=\mathrm{CH}_{3}$<smiles>[R]C(O)C(=O)NCCCCCCNC(=O)Nc1ccccc1</smiles>

Scheme-I: Synthetic route of $\alpha$-hydroxy(mercapto) amide derivatives 
$\left.\mathrm{Hz}, J_{2}=10.6 \mathrm{~Hz}, 2 \mathrm{H}, \mathrm{N}(\mathrm{CO}) \mathrm{NCH}_{2}\right), 3.04\left(\mathrm{dd}, J_{1}=6.4 \mathrm{~Hz}, J_{2}\right.$ $\left.=10.6 \mathrm{~Hz}, 2 \mathrm{H}, \mathrm{O}(\mathrm{CO}) \mathrm{NCH}_{2}\right), 6.08(\mathrm{t}, J=5.6 \mathrm{~Hz}, 1 \mathrm{H}$, $\mathrm{PhN}(\mathrm{CO}) \mathrm{NH}), 6.76(\mathrm{t}, J=5.6 \mathrm{~Hz}, 1 \mathrm{H}, \mathrm{O}(\mathrm{CO}) \mathrm{NH}), 6.85-7.37$ (m, 5H, PhH), 8.35 (s, $1 \mathrm{H}, \mathrm{PhNH})$; EI MS: $m / z(\%) 336\left([\mathrm{M}+1]^{+}\right.$, 1), 335 ( $\left.\mathrm{M}^{+}, 4\right), 279$ (10), 206 (3), 143 (12), 119 (2), 93 (100).

Preparation of 1-(6-aminohexyl)-3-phenylurea hydrochloride $(4)^{18}$ : In a $250 \mathrm{~mL}$ three neck round bottom flask, intermediate 3 (5.5 g, $0.016 \mathrm{~mol})$ and ethyl acetate $(150 \mathrm{~mL})$ were added. $\mathrm{HCl}$ (gas) was passed in reaction mixture at room temperature, the mixture was stirred for $5 \mathrm{~h}$, which was concentrated on a rotary evaporator to give white solid 4, yield $97 \%$ (4.35 g). m.p. $154-156{ }^{\circ} \mathrm{C} .{ }^{1} \mathrm{H}$ NMR $\left(400 \mathrm{MHz}, \mathrm{CDCl}_{3}\right) \delta$ (ppm): 1.32 (s, $\left.4 \mathrm{H}, \mathrm{CCCH}_{2} \mathrm{CH}_{2} \mathrm{CC}\right), 1.41$ (s, 2H, $\mathrm{NCCH}_{2}$ ), $1.55\left(\mathrm{~s}, 2 \mathrm{H},(\mathrm{CO}) \mathrm{NCCH}_{2}\right), 2.77\left(\mathrm{dd}, J_{1}=13.2 \mathrm{~Hz}, J_{2}=6.8 \mathrm{~Hz}\right.$, $\left.2 \mathrm{H}, \mathrm{NCH}_{2}\right), 3.06\left(\mathrm{dd}, J_{1}=13.2 \mathrm{~Hz}, J_{2}=6.8 \mathrm{~Hz}, 2 \mathrm{H}\right.$, (CO) $\mathrm{NCH}_{2}$ ), 6.41 (t, $\left.J=5.2 \mathrm{~Hz}, 1 \mathrm{H}, \mathrm{PhN}(\mathrm{CO}) \mathrm{NH}\right), 6.84-7.39$ (m, 5H, PhH), 7.87 (s, 2H, NH $), 8.76(\mathrm{~s}, 1 \mathrm{H}, \mathrm{PhNH})$; EI MS: $m / z(\%) 236\left([\mathrm{M}+1]^{+}, 1\right), 235\left(\mathrm{M}^{+}, 3\right), 219(1), 183(10), 149(4)$, 143(12), 93(100).

Preparation of 2-chloro-N-[6-(3-phenylureido)hexyl] acetamide $(5)^{19}$ : To a solution of intermediate $\mathbf{4}(2.0 \mathrm{~g}, 7.36$ $\mathrm{mmol})$ in $\mathrm{CH}_{2} \mathrm{Cl}_{2}(50 \mathrm{~mL})$ was added $\mathrm{Et}_{3} \mathrm{~N}(2.23 \mathrm{~g}, 22.1 \mathrm{mmol})$. The mixture was stirred at room temperature for $1 \mathrm{~h}$, then 2 chloroacetyl chloride $(1.0 \mathrm{~g}, 8.85 \mathrm{mmol})$ was dropwise added at $0{ }^{\circ} \mathrm{C}$. The resulting mixture was stirred at room temperature for $5 \mathrm{~h}$, extracted with $\mathrm{CH}_{2} \mathrm{Cl}_{2}(60 \mathrm{~mL} \times 3)$, dried with anhydrous magnesium sulfate, filtered off with suction. The combined organic layer was concentrated on a rotary evaporator, triturated with ethyl acetate $(5 \mathrm{~mL})$. The precipitate was filtrated and dried with vacuum to give white solid $\mathbf{5}$, yield $73 \%(1.67 \mathrm{~g})$. m.p. $139-141{ }^{\circ} \mathrm{C} .{ }^{1} \mathrm{H}$ NMR $\left(400 \mathrm{MHz}, \mathrm{CDCl}_{3}\right) \delta$ (ppm): 1.29 (s, $4 \mathrm{H}, \mathrm{CCCH}_{2} \mathrm{CH}_{2} \mathrm{CC}$ ), 1.43 (s, $4 \mathrm{H}, \mathrm{CCH}_{2} \mathrm{CCCH}_{2} \mathrm{C}$ ), 1.51 (d, $\left.J=6.8 \mathrm{~Hz}, 3 \mathrm{H}, \mathrm{CH}_{3}\right), 3.99\left(\mathrm{dd}, J_{1}=11.6 \mathrm{~Hz}, J_{2}=5.6 \mathrm{~Hz}, 4 \mathrm{H}\right.$, $\left.\mathrm{CH}_{2} \mathrm{CCCCCH}_{2}\right), 4.43\left(\mathrm{dd}, J_{1}=12.6 \mathrm{~Hz}, J_{2}=6.8 \mathrm{~Hz}, 1 \mathrm{H}, \mathrm{ClCH}\right)$, $6.08(\mathrm{t}, J=5.6 \mathrm{~Hz}, 1 \mathrm{H}, \mathrm{PhN}(\mathrm{CO}) \mathrm{NH}), 6.84-7.37(\mathrm{~m}, 5 \mathrm{H}$, $\mathrm{PhH}), 8.18$ (s, 1H, NH(CO)C), 8.35 (s, 1H, PhNH); EI MS: $\mathrm{m} / \mathrm{z}(\%) 313\left([\mathrm{M}+2]^{+}, 1\right), 311\left(\mathrm{M}^{+}, 3\right), 219(6), 174(12)$, 162(13), 128(21), 119(25), 106(22), 98(16), 93(100).

Preparation of 2-chloro- $\mathrm{N}$-[6-(3-phenylureido)hexyl] propanamide (6): In the same way as compound $\mathbf{5}$, intermediate compound 6 was synthesized. white solid, Yield: $71 \%$, m.p. 142-144 ${ }^{\circ} \mathrm{C} .{ }^{1} \mathrm{H}$ NMR $\left(400 \mathrm{MHz}, \mathrm{CDCl}_{3}\right) \delta(\mathrm{ppm}): 1.29$ (s, $\left.4 \mathrm{H}, \mathrm{CCCH}_{2} \mathrm{CH}_{2} \mathrm{CC}\right), 1.43$ (s, $\left.4 \mathrm{H}, \mathrm{CCH}_{2} \mathrm{CCCH}_{2} \mathrm{C}\right), 1.51(\mathrm{~d}, J=$ $\left.6.8 \mathrm{~Hz}, 3 \mathrm{H}, \mathrm{CH}_{3}\right), 3.99\left(\mathrm{dd}, J_{1}=11.6 \mathrm{~Hz}, J_{2}=5.6 \mathrm{~Hz}, 4 \mathrm{H}\right.$, $\left.\mathrm{CH}_{2} \mathrm{CCCCCH}_{2}\right), 4.43\left(\mathrm{dd}, J_{1}=12.6 \mathrm{~Hz}, J_{2}=6.8 \mathrm{~Hz}, 1 \mathrm{H}, \mathrm{ClCH}\right)$, 6.08 (t, $J=5.6 \mathrm{~Hz}, 1 \mathrm{H}, \mathrm{PhN}(\mathrm{CO}) \mathrm{NH}), 6.84-7.37$ (m, 5H, PhH), 8.18 (s, 1H, NH(CO)C), 8.35 (s, 1H, PhNH); EI MS: $m / z(\%)$ $313\left([\mathrm{M}+2]^{+}, 1\right), 311\left(\mathrm{M}^{+}, 3\right), 219(6), 174(12), 162(13)$, 128(21), 119(25), 106(22), 98(16), 93(100).

Preparation of 2-mercapto- $\mathrm{N}$-[6-(3-phenylureido)hexyl]acetamide $(7)^{20}$ : To a solution of intermediate $5(0.2 \mathrm{~g}$, $0.64 \mathrm{mmol}$ ) in $\mathrm{MeOH}$ (15 mL) was added NaSH (54 mg, 0.96 $\mathrm{mmol}$ ), the mixture was refluxed for $3 \mathrm{~h}$. The reaction mixture was cooled to room temperature, evaporated in vacuum, purified by chromatography on silica using chloroform/methanol (10:1, $\mathrm{v} / \mathrm{v})$ as eluent to give white solid 7 in yield $75 \%$ (0.15 g). m.p. 127-129 ${ }^{\circ} \mathrm{C} .{ }^{1} \mathrm{H}$ NMR (400 MHz, DMSO- $\left.d_{6}\right) \delta(\mathrm{ppm}): 1.28$ (m, $4 \mathrm{H}, \mathrm{CCCH}_{2} \mathrm{CH}_{2} \mathrm{CC}$ ), 1.41 (m, 4H, $\left.\mathrm{CCH}_{2} \mathrm{CCCH}_{2} \mathrm{C}\right), 2.72$ (t, $J=7.8 \mathrm{~Hz}, 1 \mathrm{H}, \mathrm{SH}), 3.05$ (m, 4H, $\mathrm{CH}_{2} \mathrm{CCCCCH}_{2}$ ), 3.58 (s, $\left.2 \mathrm{H}, \mathrm{CH}_{2} \mathrm{~S}\right), 6.10(\mathrm{t}, J=5.4 \mathrm{~Hz}, 1 \mathrm{H}, \mathrm{PhN}(\mathrm{CO}) \mathrm{NH}), 6.87-7.37$ (m, 5H, PhH), 7.97 (t, $J=5.4 \mathrm{~Hz}, 1 \mathrm{H}, \mathrm{NH}(\mathrm{CO}) \mathrm{C}), 8.37$ (s, $1 \mathrm{H}, \mathrm{PhNH}) .{ }^{13} \mathrm{C}$ NMR $\left(100 \mathrm{MHz}, \mathrm{DMSO}-d_{6}\right) \delta$ (ppm): 169.42, 155.20, 140.61, 128.62, 120.89, 117.51, 38.96, 38.79, 29.7, 29.0, 27.1, 26.1. LC MS calcd. 309.4, found 309.7 [M], 332.6 $[\mathrm{M}+\mathrm{Na}]^{+}$. Anal. calcd. for $\mathrm{C}_{15} \mathrm{H}_{23} \mathrm{~N}_{3} \mathrm{O}_{2} \mathrm{~S}(\%)$ : C, 58.22; $\mathrm{H}$, 7.49; N, 13.58. Found (\%): C, 58.17; H, 7.32; N, 13.37.

Preparation of 2-mercapto- $\mathrm{N}$-[6-(3-phenylureido)hexyl]propanamide (8): In the same way as compound 7, the target compound 8 was synthesized. white solid, Yield: $69 \%$, m.p. 128-130 ${ }^{\circ} \mathrm{C}$. ${ }^{1} \mathrm{H}$ NMR (400 MHz, DMSO- $d_{6}$ ) $\delta$ (ppm): 1.28 $\left(\mathrm{m}, 4 \mathrm{H}, \mathrm{CCCH}_{2} \mathrm{CH}_{2} \mathrm{CC}\right.$ ), 1.32 (d, $\left.J=6.8 \mathrm{~Hz}, 3 \mathrm{H}, \mathrm{CH}_{3}\right), 1.41$ $\left(\mathrm{m}, 4 \mathrm{H}, \mathrm{CCH}_{2} \mathrm{CCCH}_{2} \mathrm{C}\right), 2.79(\mathrm{~d}, J=8.5 \mathrm{~Hz}, 1 \mathrm{H}, \mathrm{SH}), 3.02$ $\left(\mathrm{m}, 4 \mathrm{H}, \mathrm{CH}_{2} \mathrm{CCCCCH}_{2}\right), 3.41\left(\mathrm{dd}, J_{1}=7.0 \mathrm{~Hz}, J_{2}=8.5 \mathrm{~Hz}\right.$, 1H, SCH), 6.09 (t, J = 4.0 Hz, 1H, PhN(CO)NH), 6.85-7.38 (m, 5H, PhH), 7.91 (t, $J=5.1 \mathrm{~Hz}, 1 \mathrm{H}, \mathrm{NH}(\mathrm{CO}) \mathrm{C}), 8.35$ (s, $1 \mathrm{H}, \mathrm{PhNH}) ;{ }^{13} \mathrm{C}$ NMR $\left(100 \mathrm{MHz}, \mathrm{DMSO}-d_{6}\right) \delta$ (ppm): 172.98 , 155.74, 141.13, 129.13, 121.42, 118.11, 40.08, 39.17, 36.83, $30.26,29.45,26.60,22.69$; LC-MS calcd. for $\left[\mathrm{C}_{16} \mathrm{H}_{25} \mathrm{~N}_{3} \mathrm{O}_{2} \mathrm{~S}+\right.$ $1]^{+}$, 324, found, 324.1. [2 $\left.\times \mathrm{C}_{16} \mathrm{H}_{25} \mathrm{~N}_{3} \mathrm{O}_{2} \mathrm{~S}+\mathrm{Na}\right]^{+}, 669$, found, 669.3. Anal. calcd. for $\mathrm{C}_{16} \mathrm{H}_{25} \mathrm{~N}_{3} \mathrm{O}_{2} \mathrm{~S}$ : C, 59.41; H, 7.79; N, 12.99. Found: C, 59.37; H, 7.72; N, 13.07.

Preparation of 2-hydroxy- $\mathrm{N}$-[6-(3-phenylureido)hexyl]acetamide $(\mathbf{9})^{21}$ : To a solution of $\mathbf{5}(0.5 \mathrm{~g}, 1.6 \mathrm{mmol})$ in DMF $(10 \mathrm{~mL})$ was added $\mathrm{CH}_{3} \mathrm{COONa}(0.26 \mathrm{~g}, 3.2 \mathrm{mmol})$. The mixture was stirred at $100{ }^{\circ} \mathrm{C}$ for $4 \mathrm{~h}$, cooled to room temperature and extracted with ethyl acetate $(50 \mathrm{~mL} \times 3)$, dried with anhydrous magnesium sulfate, filtered off with suction. The combined organic layer was concentrated on a rotary evaporator to give residue. Therein $\mathrm{NaOH}(0.13 \mathrm{~g}, 3.2 \mathrm{mmol}), \mathrm{H}_{2} \mathrm{O}(5 \mathrm{~mL})$, acetone $(5 \mathrm{~mL})$ were added, the mixture was stirred at room temperature for $4 \mathrm{~h}$. The resulting mixture was evaporated in vacuum to remove acetone, then $\mathrm{H}_{2} \mathrm{O}(10 \mathrm{~mL})$ was added, acidification by $\mathrm{HCl}$ to $\mathrm{pH}=3$, the precipitate was filtered and dried with vacuum to give white solid 9 in yield $80 \%(0.37 \mathrm{~g})$. m.p. 103$105{ }^{\circ} \mathrm{C} .{ }^{1} \mathrm{H}$ NMR $\left(400 \mathrm{MHz}, \mathrm{DMSO}-d_{6}\right) \delta(\mathrm{ppm}): 1.28(\mathrm{~m}$, $4 \mathrm{H}, \mathrm{CCCH}_{2} \mathrm{CH}_{2} \mathrm{CC}$ ), 1.42 (m, 4H, $\mathrm{CH}_{2} \mathrm{CCCH}_{2}$ ), 3.11 (m, 4H, $\mathrm{CH}_{2} \mathrm{CCCCCH}_{2}$ ), 3.78 (s, 2H, $\left.\mathrm{CH}_{2} \mathrm{O}\right), 5.44$ (s, 1H, OH), 6.11 (t, $J=5.6 \mathrm{~Hz}, 1 \mathrm{H}, \mathrm{PhN}(\mathrm{CO}) \mathrm{NH}), 6.85-7.23(\mathrm{~m}, 5 \mathrm{H}, \mathrm{PhH})$, 7.70 (s, 1H, NH(CO)C), 8.37 (s, 1H, PhNH). ${ }^{13} \mathrm{C}$ NMR (100 MHz, DMSO- $\left.d_{6}\right) \delta(\mathrm{ppm}): 171.54,155.18,140.54,128.58$, $120.87,117.54,61.40,38.93,37.93,29.68,29.21,26.08$. LC MS calcd. 294.3, found 294.2 [M], $316.2[\mathrm{M}+\mathrm{Na}]^{+}$. Anal. calcd. for $\mathrm{C}_{15} \mathrm{H}_{23} \mathrm{~N}_{3} \mathrm{O}_{3}: \mathrm{C}, 61.41 ; \mathrm{H}, 7.90 ; \mathrm{N}, 14.32$. Found: $\mathrm{C}$, 61.38; H, 7.82; N, 14.27.

Preparation of 2-hydroxy-N-[6-(3-phenylureido)hexyl]propanamide (10): In the same way as compound 9, the target compound 10 was synthesized. white solid, Yield: $76 \%$, m.p. 102-103 ${ }^{\circ} \mathrm{C}$. ${ }^{1} \mathrm{H}$ NMR (400 MHz, DMSO- $d_{6}$ ) $\delta$ (ppm): 1.21 $\left(\mathrm{d}, J=6.8,3 \mathrm{H}, \mathrm{CH}_{3}\right), 1.28\left(\mathrm{~m}, 4 \mathrm{H}, \mathrm{CCCH}_{2} \mathrm{CH}_{2} \mathrm{CC}\right), 1.44$ (m, $4 \mathrm{H}, \mathrm{CCH}_{2} \mathrm{CCCH}_{2} \mathrm{C}$ ), 3.08 (m, 4H, $\left.\mathrm{CH}_{2} \mathrm{CCCCCH}_{2}\right), 3.94$ (dd, $\left.J_{1}=6.0, J_{2}=12.0 \mathrm{~Hz}, 1 \mathrm{H}, \mathrm{COCH}\right), 5.42(\mathrm{~s}, 1 \mathrm{H}, \mathrm{OH}), 6.11(\mathrm{t}$, $J=5.2 \mathrm{~Hz}, 1 \mathrm{H}, \mathrm{PhN}(\mathrm{CO}) \mathrm{NH}), 6.86-7.39(\mathrm{~m}, 5 \mathrm{H}, \mathrm{PhH}), 7.67$ (s, 1H, NH(CO)C), 8.37 (s, 1H, PhNH). ${ }^{13} \mathrm{C} \mathrm{NMR} \mathrm{(100} \mathrm{MHz,}$ DMSO- $\left.d_{6}\right) \delta$ (ppm): 174.27, 155.17, 140.55, 128.58, 120.87, $117.54,67.23,38.92,38.00,29.68,29.15,26.03,21.11$. LC MS calcd. 308.4, found $308.2[\mathrm{M}], 330.2[\mathrm{M}+\mathrm{Na}]^{+}$. Anal. calcd. for $\mathrm{C}_{16} \mathrm{H}_{25} \mathrm{~N}_{3} \mathrm{O}_{3}$ : C, 62.52; $\mathrm{H}, 8.20 ; \mathrm{N}, 13.67$. Found: $\mathrm{C}$, 62.48; H, 8.15; N, 13.63. 
Assay of antitumor activities: The cytotoxic activity of synthesized $\alpha$-hydroxy (mercapto)- $N$-[6-(3-phenylureido)hexyl]amide compounds 7-10 were evaluated against a panel of human cell lines, with SAHA and 5-Fu as the reference control. The cancer cell lines were cultured in Dulbecco's modified Eagle's medium (DMEM) supplement with $10 \%$ fetal bovine serum (FBS). Approximately $4 \times 10^{3}$ cells, suspended in DMEM medium, were plated onto each well of a 96-well plate and incubated in $5 \% \mathrm{CO}_{2}$ at $37{ }^{\circ} \mathrm{C}$ for $24 \mathrm{~h}$. The subject compounds at indicated final concentrations were added to the culture medium and the cell cultures were continued for $72 \mathrm{~h}$. Fresh MTT was added to each well at a terminal concentration of $0.5 \mathrm{mg} / \mathrm{mL}$ and incubated with cells at $37^{\circ} \mathrm{C}$ for $4 \mathrm{~h}$. The formazan crystals were dissolved in $100 \mu \mathrm{L}$ DMSO each well and the absorbency at $492 \mathrm{~nm}$ (for absorbance of MTT formazan) and $630 \mathrm{~nm}$ (for the reference wavelength) was measured with the ELISA reader. All of the compounds were tested three times in each of the cell lines. The results expressed as $\mathrm{IC}_{50}$ (inhibitory concentration $50 \%$ ) were the averages of

\begin{tabular}{|c|c|c|c|}
\hline \multicolumn{4}{|c|}{$\begin{array}{l}\text { TABLE } 1 \\
\text { ANTITUMOR ACTIVITIES OF THE COMPOUNDS 7-10 } \\
\text { AGAINST THREE TUMOR CELL LINES in vitro }\end{array}$} \\
\hline \multirow{2}{*}{ Compound } & \multicolumn{3}{|c|}{$\mathrm{IC}_{50} / \mathrm{nM}$} \\
\hline & Hut 102 & MCF7 & HepG2 \\
\hline 7 & 43.6 & 28.3 & 31.2 \\
\hline 8 & 56.9 & 24.4 & 25.1 \\
\hline 9 & 57.0 & 37.1 & 29.7 \\
\hline 10 & 94.6 & 26.4 & 43.8 \\
\hline SAHA & 10.0 & 20.1 & 22.3 \\
\hline 5-Fu & $-^{\mathrm{a}}$ & 15.5 & 16.2 \\
\hline
\end{tabular}

three determinations and calculated by Bliss method. The results were illustrated in Table-1 with Sorafenib as the positive control.

\section{RESULTS AND DISCUSSION}

In the deprotection of $\mathbf{3}$, typically methods including TFA and hydrochloric acid led to inconvenient workup and low yields (26\%). However, hydrogen chloride gas gave a clean ammonium salt 4 with $97 \%$ yield. To obtain $\alpha$-mercapto- $N$ [6-(3-phenylureido)hexyl]amides, a route involved rigorous conditions using expensive triphenylmethanol, TFA and $\mathrm{Et}_{3} \mathrm{SiH}$, was reported $^{12,22}$. As outlined in Scheme-I, the mercaptopropionamide 7-8 are synthesized from 1,6-hexanediamine in this paper, obviating the need of fetid thioglycolic derivatives. There were three methods for $\alpha$-sulfhydrylation of $\alpha$-chloroamides: deacylation of acetylthio substituted intermediate ${ }^{23}$, deamidination of carbamimidothioate ${ }^{24}$ and reduction of disulfides which were obtained with sodium disulfide ${ }^{25,26}$ (Scheme-II). However, all have failed in the preparation of 7-8 after we tried the three routes. It worked well when treated with $\mathrm{NaSH}$ in refluxing methanol. Direct sulfhydrylation of our method has a superiorities of mild conditions, convenient operations and higher yields (the overall yield of 7 increase to $48 \%$ from $16 \%$ reported in literature), which provides a concise and efficient improvement. On the other hand, hydroxylations stepby-step afforded a rapid preparation of 9 or $\mathbf{1 0}$ using $\mathrm{CH}_{3} \mathrm{COONa}$ and $\mathrm{NaOH}$, subsequently.

Antitumor activity: The in vitro antitumor activities of the synthesized title compounds 7-10 against three human cancer cell lines, including Hut102, MCF7 and HepG2, were evaluated by MTT method. As shown in Table-1, the results<smiles>[R]NC(=O)C([R7])Cl</smiles><smiles>[R]NC(=O)C([R7])Cl</smiles><smiles>[R]NC(=O)C([R7])Cl</smiles>

This paper<smiles>[R]NC(=O)C([R7])Cl</smiles>

Scheme-II: Sulfhydrylation of $\alpha$-chloroamides 
indicated that title compounds 7-10 displayed a broad spectrum of antitumor activity. Although compounds 7-10 showed slightly weaker antitumor activity against Hut102, MCF7 and HepG2, they were in the same order of magnitude as the positive control. Nevertheless, replacement of the hydroxamate moieties with $\alpha$-hydroxy(mercapto) amide led to a distinct decrease in activity. It could be concluded that the hydroxamate moieties played a crucial role in the antitumor activity. Probably the hydroxamate moieties can interact much more efficiently with a Hut102 $\mathrm{Zn}^{2+}$ ion. The structural optimization of SAHA was worth to further research.

\section{Conclusion}

In summary, four $\alpha$-hydroxy(mercapto)- $N$-[6-(3-phenylureido)hexyl]amide derivatives were designed according to the strategy of bioisosteric replacement. Except 7 the other title compounds were newly synthesized. The titled compounds 7-10 were synthesized through a novel synthetic procedure with wild conditions and acceptable yields. Direct sulfhydrylation using $\mathrm{NaSH}$ from $\alpha$-chloroamide will become an extremely useful synthetic method for $\alpha$-mercaptoamides. The preliminary bioassay showed that all of the target compounds exhibited certain antitumor activities against Hut102, MCF7 and HepG2 cell lines.

\section{ACKNOWLEDGEMENTS}

This work was supported by Project supported by the Planned Science and Technology Project of Hunan Province, China (No. 2012NK3098) and Scientific Research Fund of Hunan Provincial Education Department of China (No. 11A092).

\section{REFERENCES}

1. P.A. Marks, R.A. Rifkind, V.M. Richon, R. Breslow, T. Miller and W.K. Kelly, Nat. Rev. Cancer, 1, 194 (2001).

2. A. Vaisburg, I. Paquin, N. Bernstein, S. Frechette, F. Gaudette, S. Leit, O. Moradei, S. Raeppel, N. Zhou, G. Bouchain, S.H. Woo, Z. Jin, J. Gillespie, J. Wang, M. Fournel, P.T. Yan, M.-C. Trachy-Bourget, M.-F. Robert, A. Lu, J. Yuk, J. Rahil, A.R. MacLeod, J.M. Besterman, Z. Li and D. Delorme, Bioorg. Med. Chem. Lett., 17, 6729 (2007).

3. D.C. Drummond, G.K. Noble, D.B. Kirpotin, Z. Guo, G.K. Scott and C.C. Benz, Annu. Rev. Pharmacol. Toxicol., 45, 495 (2005).
4. M.F. Fraga, E. Ballestar, A. Villar-Garea, M. Boix-Chornet, J. Espada, G. Schotta, T. Bonaldi, C. Haydon, S. Ropero, K. Petrie, N.G. Iyer, A. Pérez-Rosado, E. Calvo, J.A. Lopez, A. Cano, M.J. Calasanz, D. Colomer, M.Á. Piris, N. Ahn, A. Imhof, C. Caldas, T. Jenuwein and M. Esteller, Nat. Genet., 37, 391 (2005).

5. C.M. Marson, T. Mahadevan, J. Dines, S. Sengmany, J.M. Morrell, J.P. Alao, S.P. Joel, D.M. Vigushin and R.C. Coombes, Bioorg. Med. Chem. Lett., 17, 136 (2007).

6. J.E. Bolden, M.J. Peart and R.W. Johnstone, Nat. Rev. Drug Discov., 5, 769 (2006).

7. T. Hideshima, J.E. Bradner, J. Wong, D. Chauhan, P. Richardson, S.L. Schreiber and K.C. Anderson, Proc. Natl. Acad. Sci. USA, 102, 8567 (2005).

8. M. Yoshida, S. Horinouchi and T. Beppu, Bioessays, 17, 423 (1995).

9. H. Rajak, A. Agarawal, P. Parmar, B.S. Thakur, R. Veerasamy, P.C. Sharma and M.D. Kharya, Bioorg. Med. Chem. Lett., 21, 5735 (2011).

10. T.A. Miller, D.J. Witter and S. Belvedere, J. Med. Chem., 46, 5097 (2003).

11. P.C. Chen, V. Patil, W. Guerrant, P. Green and A.K. Oyelere, Bioorg. Med. Chem., 16, 4839 (2008)

12. A.P. Kozikowski, Y.F. Chen, A. Gaysin, B. Chen, M.A. D'Annibale, C.M. Suto and B.C. Langley, J. Med. Chem., 50, 3054 (2007).

13. T. Suzuki, S. Hisakawa, Y. Itoh, T. Suzuki, S. Hisakawa, N. Suzuki, K. Takahashi, M. Kawahata, K. Yamaguchi, H. Nakagawa and N. Miyata, Bioorg. Med. Chem. Lett., 17, 4208 (2007).

14. K. Dodo, T. Minato and Y. Hashimoto, Chem. Pharm. Bull. (Tokyo), 57, 190 (2009)

15. J.J. Diaz-Mochon, M.A. Fara, R.M. Sanchez-Martin and M. Bradley, Tetrahedron Lett., 49, 923 (2008).

16. W. Zhang, C.H.T. Chen and T. Nagashima, Tetrahedron Lett., 44, 2065 (2003).

17. J. Scheerder, J.F.J. Engbersen, A. Casnati, R. Ungaro and D.N. Reinhoudt, J. Org. Chem., 60, 6448 (1995).

18. M.A. Cinelli, B. Cordero, T.S. Dexheimer, Y. Pommier and M. Cushman, Bioorg. Med. Chem., 17, 7145 (2009).

19. M. Somei, Y. Fukui, M. Hasegawa, N. Oshikiri and T. Hayashi, Heterocycles, 53, 1725 (2000).

20. S. Paramasivam, A. Balakrishnan, O. Dmitrenko, A. Godert, T.P. Begley, F. Jordan and T. Polenova, J. Phys. Chem. B, 115, 730 (2011).

21. S.P. Breazzano and D.L. Boger, J. Am. Chem. Soc., 133, 18495 (2011).

22. B. Chen, P.A. Petukhov, M. Jung, A. Velena, E. Eliseeva, A. Dritschilo and A.P. Kozikowski, Bioorg. Med. Chem. Lett., 15, 1389 (2005).

23. K.J.C. van Bommel, M.R. de Jong, G.A. Metselaar, W. Verboom, J. Huskens, R. Hulst, H. Kooijman, A.L. Spek and D.N. Reinhoudt, Chem. Eur. J., 7, 3603 (2001).

24. A.M.A. Muhanna, E. Ortiz-Salmerón, L. Garcia-Fuentes, J.J. GiménezMartínez and A. Vargas-Berenguel, Tetrahedron Lett., 44, 6125 (2003).

25. Y.C. Luo, H. Zhang and Y. Yang, Jingxi Huagong Zhongjianti, 37, 43 (2007).

26. R.A. Aitken, D.P. Armstrong, R.H.B. Galt and S.T.E. Mesher, J. Chem. Soc., Perkin Trans. I, 935 (1997). 